DISTRIBUTION STATEMENT A. Approved for public release; distribution is unlimited.

\title{
Comparative and Cumulative Energetic Costs of Odontocete Responses to Anthropogenic Disturbance
}

\author{
Marla M. Holt \& Dawn P. Noren \\ NOAA NMFS Northwest Fisheries Science Center \\ 2725 Montlake Blvd. East \\ Seattle, WA 98112 \\ phone: (206) 860-3261 fax: (206) 860-3475 email: marla.holt@noaa.gov \\ phone: (206) 302-2439 fax:(206) 860-3475 email: dawn.noren@noaa.gov \\ Robin Dunkin \& Terrie M. Williams \\ Ecology and Evolutionary Biology Dept. \\ University of California, Santa Cruz \\ 100 Shaffer Road \\ Santa Cruz, CA 95060 \\ phone: (831) 334-0640 fax: (831) 459-3383 email: dunkin@biology.ucsc.edu \\ phone: (831) 459-5123 fax: (831)459-3383 email: williams@biology.ucsc.edu \\ Award Number: N0001414IP20045 / N000141440460 \\ http://www.nwfsc.noaa.gov/research/divisions/cbd/marine_mammal/marinemammal.cfm
}

\section{LONG-TERM GOALS}

Cetacean responses to marine anthropogenic activities include changes in acoustic behavior, surface active behavior, dive behavior, direction of travel, and behavioral activity states. Behavioral effects have been observed in several field studies involving both observational and controlled exposure experiments. However, the consequences of these behavioral responses are often difficult to quantify in biological currencies. Previous work has empirically measured the energetic consequences of many of these behavioral responses that may have acute or chronic energetic consequences including our ONR-supported work on the metabolic costs of communicative sound and click production, and the metabolic costs of changes in vocal behavior in bottlenose dolphins that are consistent with noiseinduced vocal modification. The current investigation involves two separate but related studies that will address energetic costs of behavioral responses to anthropogenic disturbance, including acoustic effects, in odontocetes. The first study will address, in a comparative framework, metabolic costs of sound production and vocal modification across different sound types and odontocete species. The second component of the investigation will address cumulative energetic costs of behavioral responses to disturbance. These analyses will provide quantitative information that will be particularly useful to incorporate into models such as the Population Consequence of (Acoustic) Disturbance (PCAD/PCoD) as well as provide input data for environmental assessments/impact statements and other permit processes involving anthropogenic activities that have the potential to impact marine mammals. 


\section{Report Documentation Page}

Form Approved

OMB No. 0704-0188

Public reporting burden for the collection of information is estimated to average 1 hour per response, including the time for reviewing instructions, searching existing data sources, gathering and maintaining the data needed, and completing and reviewing the collection of information. Send comments regarding this burden estimate or any other aspect of this collection of information,

including suggestions for reducing this burden, to Washington Headquarters Services, Directorate for Information Operations and Reports, 1215 Jefferson Davis Highway, Suite 1204, Arlington

VA 22202-4302. Respondents should be aware that notwithstanding any other provision of law, no person shall be subject to a penalty for failing to comply with a collection of information if it

does not display a currently valid OMB control number.

1. REPORT DATE

30 SEP 2014

4. TITLE AND SUBTITLE

Comparative and Cumulative Energetic Costs of Odontocete Responses to Anthropogenic Disturbance

6. AUTHOR(S)

7. PERFORMING ORGANIZATION NAME(S) AND ADDRESS(ES)

National Oceanic and Atmospheric Administration (NOAA), NMFS

Northwest Fisheries Science Center,2725 Montlake Blvd.

East,Seattle,WA,98112

9. SPONSORING/MONITORING AGENCY NAME(S) AND ADDRESS(ES)
3. DATES COVERED

00-00-2014 to 00-00-2014

5a. CONTRACT NUMBER

5b. GRANT NUMBER

5c. PROGRAM ELEMENT NUMBER

5d. PROJECT NUMBER

5e. TASK NUMBER

5f. WORK UNIT NUMBER

8. PERFORMING ORGANIZATION

REPORT NUMBER

10. SPONSOR/MONITOR'S ACRONYM(S)

11. SPONSOR/MONITOR'S REPORT NUMBER(S)

12. DISTRIBUTION/AVAILABILITY STATEMENT

Approved for public release; distribution unlimited

13. SUPPLEMENTARY NOTES

14. ABSTRACT

15. SUBJECT TERMS

16. SECURITY CLASSIFICATION OF:

a. REPORT

unclassified b. ABSTRACT

unclassified c. THIS PAGE

unclassified
17. LIMITATION OF ABSTRACT

Same as

Report (SAR)
18. NUMBER 19a. NAME OF OF PAGES

5 


\section{OBJECTIVES}

The specific goals of the first year (beginning in FY2014) of this investigation include the following: (1) initiate the comparison of the metabolic cost of whistle and click sound production in bottlenose dolphins using previously collected empirical data to determine if distinct sound types confer different energetic costs to the signaler, and (2) initiate the development of a predictive bioenergetic model of the metabolic costs of sound production across several cetacean species from the determination of the mass of sound producing muscles in bottlenose dolphins and other species. Select biochemical analyses of these muscles will also be conducted to better assess their aerobic and anaerobic capacities and determine whether there are any differences in capacity across species. This phase of our work is ongoing and will continue through the end of the 2014 calendar year.

In subsequent years (Year 2 and 3), the comparison of the energetic cost of whistle and click sound production and the cost of sound production bioenergetic model will be completed, the estimates of cumulative impacts will be initiated and completed, and results will be presented at professional conferences and submitted for peer-reviewed publication.

\section{APPROACH}

Odontocetes produce a wide variety of sounds known to have distinct signal qualities and functionalities. Tonal sounds, such as whistles, function as communicative sounds while broadband clicks are used for echolocating on prey in bottlenose dolphins. Whistles are much longer in duration and require close to twice the nasal air pressure to produce relative to the generation of echolocation clicks (Ridgway et al. 2001; Cranford et al., 2011). Thus, it is predicted that whistle production is energetically more costly than click production but this prediction is complicated by the fact that clicks are generally produced at much higher intensities. Our previous work has measured the energetic cost associated with production of these distinct sound types: whistles and squawks used for communicative purposes (Noren et al. 2013) and clicks used for echolocation (see final MBholt report on award no. N0001412IP20072, Noren et al. in prep). A key finding from previous work is that the metabolic cost (relative to resting) of social sound production is related to the energy content of the sounds produced. More recently, we have found that metabolic rates during click generation are also related to the energy content of the clicks but, consistent with field studies, the average click energy produced on a per trial basis was higher than that for social sound production trials. In contrast to whistle/squawk production trials, increases in metabolic rate (relative to resting) does not appear to extend beyond the period in which the dolphin is actively clicking. In order to the assess differences in the metabolic costs of click and whistle/squawk production, comparisons should be made for equal energy sound generation. The relationships between metabolic rates and the vocal effort (quantified as the energy content of the sounds produced) derived from our previous empirical investigations will allow such comparisons.

Previous studies in vertebrates have found that sound production muscles are physiologically and anatomically specialized to facilitate a high contraction frequency (e.g., Elemans et al. 2011). Because energy is required to facilitate each contraction, these muscles are among the most energetic of all vertebrate skeletal muscle. The amount of sound-producing muscle mass in relation to body mass is also a good predictor of the metabolic cost of sound production in a variety of animal taxa (Ophir et al. 2010). In the current study, the mass of sound-producing muscles relative to total body size will be determined in a variety of odontocete cetaceans of different body sizes (e.g. harbor porpoise, bottlenose dolphin, killer whale, beaked whale, as available) through a collaborative effort among 
researchers at NOAA, UC Santa Cruz, Dr. Ted Cranford at Quantitative Morphology Consulting, Inc., and Maureen (Moe) Flannery at the California Academy of Sciences. These data will then be incorporated into a bioenergetic model of sound production for odontocete cetaceans. Computed tomography (CT) scans of the head and upper thorax of recently deceased stranded odontocetes will be used to determine the volume of sound-producing muscle mass for each individual. Specimens must be fresh dead (stranding code 2) and in robust body condition prior to scanning in order to be included in the data set. As applied previously in humans (Dériaz et al. 1992), segmentation analysis of the major muscle groups involved in sound production (Ridgway et al. 1980, Cranford et al., 2011) will be performed to delineate these groups based on differences in electron density from the surrounding tissue. Muscle volume will be determined from integration of the segmented area and total muscle mass determined from this and values of muscle density. To the extent possible, densities (mass volume $^{-1}$ ) of samples from these muscle groups will be empirically measured. Myoglobin content, acid buffering capacity, and muscle histochemistry (e.g., fiber typing, capillary density, and/or enyzmatic properties ) of the sound producing muscles from different odontocete species will be assessed to provide further details on the physiological performance and potential interspecific differences in the energetic demands of vocal activity. Anatomical dissection on representative individuals, as available, will also be performed to validate estimated muscle mass determined from CT scan data.

The second component of the investigation will address cumulative energetic costs of behavioral responses to disturbance (to be initiated in Year 2). Energetic impact of disturbance scenarios will be estimated and scaled as appropriate for each of the scenarios using published empirical data describing behavioral responses and empirically measured behavior-specific energetic costs. Scenarios will include different sound exposure types (e.g. sonar, vessel noise), level of exposure (e.g. duration, intensity), and magnitude of the response (types of behavior and physiologic/energetic effects). Metabolic costs will be reported in units that can be related to an individual odontocete's activity budget and daily prey energy requirements.

\section{WORK COMPLETED}

From July 2014 (when FY2014 funds were obtained) to September 2014, the following work has been completed for the study. Comparisons of the metabolic costs of distinct sound types (social sounds and click sounds) in bottlenose dolphins have been initiated. Preliminary results are described in the next section. We have also received all of the currently available CT scan data from Cal Academy and have preliminarily identified which scans, from over 25 available, are viable candidates. These are available for segmentation analysis as soon as the contract with UCSC and Quantitative Morphology Consulting, Inc is finalized. We have recruited Dr. Joy Reidenberg to assist in a detailed head dissection, scheduled at UCSC in early December 2014, to help develop a muscle collection protocol and to ground truth our CT scan data. The final collection protocol for the muscle histochemistry will be written up and distributed after the dissection session. One porpoise (Phocoena phocoena) has been collected for the head dissection. Request for additional specimens to be collected for head dissections and muscle histochemistry work has been made to the west coast stranding network. All supplies for muscle myoglobin and acid buffering analyses have been ordered and received and the laboratory space to run these analyses has been set up at UCSC. Protocols, supplies, and laboratory set-up for muscle histochemistry (e.g., fiber typing, and/or capillary density, and/or enyzmatic properties) will be completed after the December dissection session. 


\section{RESULTS}

Because FY2014 funding was received late in the fiscal year and we are just getting started on the project, results are limited. The comparison of the metabolic costs between sound types in bottlenose dolphins should be considered preliminary. Differences in methodologies and the energy level of the sounds produced by subjects have been considered for the comparison. Because clicks were produced while submerged, the average metabolic rate during submerged silence (measured on control trials) was subtracted from the average metabolic rate during submerged clicking to isolate the average metabolic cost of click production for each of the two dolphin subjects. The isolated cost of producing continuous clicks for 2.25 min was 0.4 and $0.7 \mathrm{kcal}$ for Dolphin A and B, respectively. The higher cost of producing continuous clicks for Dolphin B, again, was likely related to the higher average energy level of the clicks he produced (average cSEL $=160 \mathrm{~dB}$ re $1 \mu \mathrm{Pa}^{2} \mathrm{~s}$ ) relative to Dolphin A (average cSEL $=154 \mathrm{~dB}$ re $1 \mu \mathrm{Pa}^{2} \mathrm{~s}$ ). Using the empirically derived relationship between total metabolic cost and vocal effort for communicative sound production in Dolphin B (Holt et al. in review), the average metabolic cost in kcals for producing 2.25 min of squawks at cSEL of $160 \mathrm{~dB}$ re 1 $\mu \mathrm{Pa}^{2} \mathrm{~s}$ is predicted to be $13 \mathrm{kcals}$, respectively. The estimated energetic costs of producing these sound types can easily be related to quantities of prey and daily energy budgets. However, these estimates are for vocal behavior at fairly high repetition rates so more realistic comparisons should be made relative to the natural behavior of free-ranging dolphins. For example, the performed repetition rates during click production were similar to field measurements in bottlenose dolphins but repetition rates of social sounds were much higher compared to those typically observed in the field. Nonetheless, preliminary results indicate that the metabolic cost of click production appears to be much smaller than the metabolic cost of communicative signal production of equal energy content in bottlenose dolphins.

\section{IMPACT/APPLICATIONS}

Results of this investigation will provide important quantitative data that can be incorporated into efforts to assess the biological significance of behavioral and acoustic responses to anthropogenic sound exposure including activites with naval relevance.

\section{RELATED PROJECTS}

Dr. Terrie Williams' laboratory is conducting other studies related to this project. The goal of one related study is to assess the physiological costs and potential risks of common responses by cetaceans to anthropogenic noise. http://williams.eeb.ucsc.edu/

\section{REFERENCES}

Cranford, T. W., Elsberry, W. R., Van Bonn, W. G., Jeffress, J. A., Chaplin, M. S., Blackwood, D. J., Carder, D. A., Kamolnick, T., Todd, M. A., and Ridgway, S. H. 2011. Observation and analysis of sonar signal generation in the bottlenose dolphin (Tursiops truncatus): Evidence for two sonar sources. Journal of Experimental Marine Biology and Ecology 407, 81-96.

Dériaz, O., Fournier, G., Tremblay, A., Després, J. P., and Bouchard, C. 1992. Lean-body-mass composition and resting energy expenditure before and after long-term overfeeding. The American Journal of Clinical Nutrition 56, 840-847. 
Elemans, C. P. H., Mead, A. F., Jakobsen, L., and Ratcliffe, J. M. 2011. Superfast muscles set maximum call rate in echolocating bats. Science 333, 1885-1888.

Noren, D.P., Holt, M.M., Dunkin, R.C., and Williams, T.M. 2013. The metabolic cost of communicative sound production in bottlenose dolphins (Tursiops truncatus). Journal of Experimental Biology 216, 1624-1629

Ophir, A. G., Schrader, S. B., and Gillooly, J. F. 2010. Energetic cost of calling: general constraints and species-specific differences. Journal of Evolutionary Biology 23, 1564-1569.

Ridgway, S. H., Carder, D. A., and Green, R. F. 1980. Electromyographic and pressure events in the nasolaryngeal system of dolphins during sound production. In R.G. Busnel and J.F. Fish (Eds.), Animal Sonar Systems, Plenum Publishing Corporation, New York, pp. 239-250. 